\title{
LAS TECNOLOGÍAS DE LA INFORMACIÓN Y LA COMUNICACIÓN EN LA EXPERIENCIA DE LOS VIAJEROS MILLENNIALS RESIDENTES DE TIJUANA
}

\author{
ANa María MiRanda-ZaVala \\ amiranda@uabc.edu.mx \\ ISAAC CRUZ-ESTRADA \\ icruz@uabc.edu.mx \\ MARGARITA RAMÍREZ-TORRES \\ mramirez@uabc.edu.mx \\ Universidad Autónoma de Baja California
}

En la investigación se estudia la experiencia del viajero con perfil millennial residente de la ciudad de Tijuana en el uso de las Tecnologías de Información y la Comunicación (TIC) durante su visita a destinos turísticos. Es un estudio de corte cuantitativo; se aplicó un cuestionario a 385 millennials residentes de Tijuana, mediante un muestreo aleatorio, con base en $95 \%$ de confianza y $5 \%$ error admitido. Los participantes viajaron por lo menos cinco veces fuera del estado de Baja California en el 2019. Asimismo, se realizó un análisis factorial para conocer los servicios digitales que utiliza dicho sector de la población. Como resultados los servicios tecnológicos usados con mayor frecuencia en el destino son: geolocalización, que optimiza tiempos del recorrido; comparten experiencias y realizan recomendaciones mediante redes sociales; buscan información en sitios web y redes sociales de los negocios turísticos. Las apps que más usan son: Facebook, Instagram, WhatsApp y Uber. Se concluye que los servicios tecnológicos favorecen la experiencia del viajero con ese perfil, en este sentido las TIC resultan ser componentes claves dentro de los elementos que hacen a un destino competitivo.

Palabras clave: viajeros, millennials, TIC, innovación turística, Tijuana.

\section{ICT IN THE EXPERIENCE OF MILLENNIAL TRAVELERS LIVING IN TIJUANA}

This research studies the experience of the traveler with a millennial profile living in the city of Tijuana in the use of Information and Communication Technologies (ICT) during the visit to tourist destinations. It is a quantitative study. A questionnaire was applied to 385 millennials residents of Tijuana, through a random sampling, based on $95 \%$ confidence and $5 \%$ admitted error. The participants traveled at least five times outside the state of Baja California in 2019. Likewise, a factor analysis was carried out to find out the digital services used by said sector of the population. As a result, the most frequently used tech-nological services in the destination are: geolocation, which optimizes travel times; share experiences and make recommendations through social networks; They look for information on websites and social networks of tourism businesses. The apps they use the most are: Facebook, Instagram, WhatsApp and Uber. It is concluded that technological services favor the experience of the traveler with this profile, in this sense ICTs turn out to be key components within the elements that make a competitive destination.

Keywords: travelers, millennials, ICT, tourism innovation, Tijuana. 


\section{Introducción}

a revolución de las Tecnologías de Información y Comunicación (TIC) y su impacto en niños y jóvenes han provocado la necesidad de acceso a grandes volúmenes de información disponibles en la web (Caballero y Baigorri, 2013). Asimismo, la transición hacia una sociedad digital ha venido acompañada en un principio por el acceso al correo electrónico, herramientas de mensajería instantánea, las redes sociales, videojuegos y otros dispositivos conectados a internet, logrando en estos últimos años una atención especial en el teléfono móvil, el cual se ha convertido en un elemento indispensable y una extensión necesaria en la vida de las personas (Díaz-Sarmiento et al., 2017).

Con el aumento exponencial al uso de herramientas y plataformas tecnológicas, aparecen otros nichos de mercado, los cuales se han vuelto consumidores y productores de contenido a la vez, este rol ha impulsado el trabajo colaborativo en la red más allá de los intereses personales (Feixa et al., 2016). En este caso de estudio se analiza a la generación $Y$, también conocida como millennials, formada por personas nacidas entre los años 1981 y 2000 que crecieron en una época de grandes avances tecnológicos, lo cual ha conducido a una relación intensiva y fascinación por utilizar los medios digitales para disponer de los servicios de empresas (Carvalho et al., 2015; Díaz-Sarmiento et al., 2017).

La población que conforma este segmento se constituye sustancialmente a partir de la expansión de internet y la globalización de los mercados, se pronuncia con tener una conectividad a escala mundial, con la necesidad de acceso e inmediatez a la información (Garzaniti, 2018). Los millennials tienen a disposición diversas fuentes de información con una conexión global, empleadas para comparar y tomar decisiones en su vida cotidiana (Carvalho et al., 2015). No obstante, para el Organismo Internacional de Juventud (OIJ, 2017) y Ruiz-Cartagena (2017) existe heterogeneidad en esta generación, con dos sectores que se le atribuyen: 1) los nativos digitales, en los que se expresa la revolución digital, la velocidad extrema de información $y$ nuevas formas de pensar; $y b$ ) el otro sector que se ha hecho adulto con el cambio de siglo, que se considera una generación bisagra entre lo viejo y lo nuevo. Por tanto, este trabajo se enfocó en los nativos digitales debido a que se consideran personas tecnológicas.

En cuanto al comportamiento dentro del turismo, los perfiles millennial buscan formas peculiares de entretenimiento, con propósitos gratificantes y significados en 
las experiencias que desean obtener; en su mayoría planifican sus viajes usando recursos tecnológicos, lo que también contempla diferentes hábitos de compra en comparación a las generaciones que los anteceden (Ordaz-Mejía y Osorio-García, 2016). Ellos usan las diversas aplicaciones disponibles en la web como sus principales medios de información y comunicación con las organizaciones turísticas, aprovechan la disponibilidad de la tecnología para la compra de los servicios disponibles en el destino; además, se distinguen por personalizar su desplazamiento para optimizar el tiempo y hacer más eficiente su travesía (Castro et al., 2018).

Cabe destacar que la innovación tecnológica se ha vuelto una necesidad en la industria del turismo, tomando en cuenta las oportunidades que representa para conectar a las personas con los atractivos naturales y culturales de cada región del mundo (López-Rodríguez y López-Rodríguez, 2018). Para las personas con este perfil la tecnología se ha vuelto un componente importante en la experiencia de viaje, ya que desde niños han tenido la oportunidad de utilizar internet para organizar sus travesías y buscar ofertas, con el propósito de obtener servicios a la medida (Macanás-López, 2019).

Como se señaló previamente, para propósitos de esta investigación se estudia a los millennials considerados nativos digitales, analizando la experiencia de los viajeros de esta generación residentes en la ciudad de Tijuana respecto al uso de las tecnologías desde la planeación y durante su estancia en los destinos turísticos visitados. Los participantes en la investigación confirmaron haber realizado al menos cinco viajes fuera del estado de Baja California en el año 2019.

\section{Revisión de la literatura}

Mediante las TIC es posible motivar el desplazamiento turístico, recomendando las mejores opciones para los visitantes (Castro et al., 2018); esto crea oportunidades para que las organizaciones integren las tecnologías necesarias en su estructura empresarial en los procesos de gestión, la mercadotecnia y en la comunicación, con apoyo de los gobiernos locales y federales, que coincidan en lograr beneficios para la industria y los residentes de estas comunidades (López-Rodríguez y López-Rodríguez, 2018).

Los millennials en la actividad turística propician el estudio de los diferentes elementos que motivan a este segmento de la población, el cual se caracteriza por tener un consumo poco descifrable, producto de la globalización, la digitalización 
de la sociedad y el conocimiento, suscitando cambios radicales y bruscos en la industria del turismo, impulsando la actualización constante de la oferta y la comunicación con los viajeros (Posso y Cardona, 2016).

Dichos perfiles emplean las TIC a su favor, para facilitar la gestión del viaje, satisfacer sus necesidades durante el mismo y potenciar sus experiencias turísticas (Castro et al., 2018). El entorno de estos primeros nativos digitales siempre ha estado rodeado de cierta tecnología, siendo parte de su estilo de vida (OIJ, 2017).

Por lo anterior, es importante conocer los servicios que están siendo utilizados por los viajeros, los cuales se transforman en canales de interés para hacer llegar la oferta a las personas interesadas en viajar en la siguiente oportunidad (Orgaz y Moral, 2016). Además, se requiere descubrir los factores tecnológicos que influyen en los turistas, su efectividad en la decisión de la compra y las características distintivas de los lugares que son de su interés, poniendo especial atención en el comportamiento de la generación millennial (Posso y Cardona, 2016), tomando en cuenta las condiciones de digitalización presentes desde su niñez.

\subsection{Experiencia turística}

Toda experiencia humana, sucede a nivel cerebral, lo que se percibe mediante los sentidos es procesado por impulsos químico-eléctricos los cuales son producidos en la comunicación neuronal, esto implica que toda experiencia de la realidad se produce al interior de cada individuo (González-Damián, 2018). Este acontecimiento personal asume un significado emocional importante, que se basa en la interacción con los estímulos que son productos o servicios consumidos (Holbrook y Hirschman, 1982), pudiendo provocar experiencias extraordinarias en la vida (Arnould y Price, 1993).

Así, la experiencia turística puede definirse como una evaluación subjetiva que tiene cada persona y las expectativas presupuestadas (afectiva, cognitiva y de comportamiento) con base en los acontecimientos, los cuales se relacionan con las actividades turísticas antes (planificación), durante (en el destino) y después del viaje, añadiéndose como un recuerdo (Tung y Brent, 2011). La experiencia que obtiene el individuo durante un viaje no puede caracterizarse como una sola, sino como un conjunto de diversas situaciones, algunas de ellas obtenidas como turista y otras no (González-Damián, 2018). Cabe destacar que las organizaciones y proveedores de servicios en el destino procuran facilitar el desarrollo de un entorno propicio, 
orientado a aumentar las posibilidades de que las personas puedan transformar sus experiencias en algo memorable (Carballo-Fuentes, 2014).

En este contexto los turistas no se satisfacen fácilmente con un evento oficial artificial, sino que buscan auténticas experiencias (MacCannell, 1973). Las personas recrean los momentos significativos, involucrándose más de lo que normalmente se espera, lo que provoca en el futuro el relato de sus viajes, en donde comparten sentimientos y sensaciones de descubrimiento durante sus travesías (Harrison, 2001). Al recordar esos instantes, el turista busca espacios en los que pueda comunicar esta información, compuesta de circunstancias satisfactorias, que ameritan -desde su perspectiva- ser compartidas con las personas que forman parte de su entono, con el propósito de distinguirse y hacer que otros imiten estas experiencias (Carballo-Fuentes, 2014).

Asimismo, la experiencia está relacionada con la emoción; el turista desea descubrir, disfrutar y conectar con la gente del lugar y sus costumbres, quiere remontarse a su lugar de origen con una historia vivida, con nuevas emociones (Mazarrasa, 2016). El éxito de la oferta de experiencia en el destino se basa en que sea auténtica, mediante características diferenciales del patrimonio, paisaje, cultura y las poblaciones (Rivera, 2013; Mazarrasa, 2016).

La tecnología en la actualidad está permitiendo a los viajeros disponer de información de todo tipo sobre los lugares que son de su interés, los turistas se han vuelto más exigentes y actúan de forma interactiva, con el propósito de encontrar un tipo de viaje y actividad distinto al de masas, reclamando productos diferentes, destinos alternativos y servicios hechos a la medida que formen parte de su experiencia (Rivera, 2013).

En la industria turística, el viajero del siglo XXI toma las decisiones respecto al destino a visitar y las empresas a contratar durante sus travesías, de acuerdo con las experiencias que desea vivir, no necesariamente de acuerdo con la calidad de los servicios que espera recibir, sino en la esperanza de la experiencia turística (González-Damián, 2018). Los turistas de la generación millennial asumen los viajes como una opción para alcanzar el desarrollo personal y contribuir al desenvolvimiento social (Ordaz-Mejía y Osorio-García, 2016). Esto impulsa a los jóvenes a buscar nuevas rutas de interés, turismo alternativo, actividades de aventura y visitas a sitios donde obtengan recuerdos emblemáticos (He et al., 2014; Posso y Cardona, 2016). 
Los turistas de esta generación buscan nuevas experiencias y estilos de viaje, que les permitan sentirse realizados y satisfechos con cada situación vivida durante su travesía en el destino turístico seleccionado (Posso y Cardona, 2016). De tal manera, el futuro de los viajes es la búsqueda de aventura por parte de las personas y la tecnología tiene un lugar importante que contribuye en la experiencia turística antes, durante y después del viaje (Amadeus, 2015).

\subsection{Servicios tecnológicos que contribuyen en la experiencia del turista}

Desde hace algunas décadas las TIC y el turismo van de la mano (Neuhofer et al., 2013); a partir de 1980 (Ivars et al., 2016) las TIC y el surgimiento del internet han favorecido importantes cambios en los procesos y en el consumo en este sector. Las TIC han cambiado los paradigmas y eliminado las fronteras físicas, parte de la actividad económica realizada con estas herramientas tiene lugar entre individuos de diferentes países (López-Rodríguez y López-Rodríguez, 2018).

Así, desde la década de los 80 las tecnologías han transformado al turismo en lo que corresponde a prácticas de negocios y su estructura, esto ha contribuido a que se conviertan en una ventaja competitiva (Rodríguez-Moreno, 2018). La generación de los millennial surgió con el auge de internet, el cual ha jugado un papel decisivo en la globalización (Ulloa, 2018), por lo que la conectividad tecnológica es central en la vida en este segmento de la población (OIJ, 2017).

Las tecnologías han facilitado el acceso a información turística para los consumidores, reconfigurando la distribución de los servicios y potenciándola más allá de los resultados obtenidos en los canales tradicionales de difusión (Vieira et al., 2017). Los consumidores actuales de este sector requieren encontrar información fiable, con acceso sencillo y flexible para obtener lo que desean, por este motivo resulta fundamental mantener actualizado y en congruencia lo publicado tanto en el sitio web principal y redes sociales empleadas por las compañías, para compartir su mensaje publicitario y de promoción (Melián y Bulchand, 2015). En cuanto a los cambios en la forma de viajar de los turistas con perfil millennial, estos aprovechan la interactividad mediante diversos canales de comunicación digital, en la atención de las necesidades y las expectativas de las nuevas generaciones de viajeros (Ralph, 2017).

Ballesteros et al. (2014) y Chang y Shen (2018) señalan que la tecnología se ha convertido en un factor que contribuye a la experiencia y a la satisfacción de los con- 
sumidores, al momento de realizar la compra de los servicios. El uso de las tecnologías durante el viaje se hace cada vez más imprescindible como herramienta en la planeación, durante y después del viaje, siendo esta última etapa en la que los individuos comparten algunos de los acontecimientos memorables, a través de las diversas plataformas existentes en internet (González-Damián, 2018).

El estilo de vida de los millennials está provocando grandes trasformaciones en la industria turística, considerando que la globalización y la tecnología forman parte de su vida (Villar-Lama, 2018). El perfil turístico de estas personas es la búsqueda de precios competitivos y reservaciones mediante su smartphone, además de múltiples consultas en otras fuentes de información en línea antes de comprar, así como la importancia que le otorgan a los comentarios de otros viajeros en redes sociales, blogs, páginas web, etcétera. Sus gastos se centran más en la comunidad local, el valor de sus experiencias y durante todo su viaje, desde el inicio hasta el final, están conectados a internet (Villar-Lama, 2018; Gustavo, 2013; Organización Mundial del Turismo, OMT, 2016).

En ese sentido, las ventas digitales han propiciado la inmediatez, facilitando el acceso a la compra, convirtiéndose en un factor que impulsa a la profesionalización de los servicios tecnológicos, los cuales garanticen y ofrezcan seguridad a los turistas, quienes confían en realizar su reservación de hospedaje, transporte y recorridos a través de estos canales (Castro et al., 2018). Con ello, las TIC en el mercado turístico contemplan una tendencia positiva (Cerezo y Guevara, 2015).

\subsection{Comportamiento del turista de la generación millennial}

El turismo que realizan representa un nicho de mercado importante y atractivo para la industria turística, producto de un comportamiento heterogéneo que para los mayores de 40 años podría entenderse como liberal (Posso y Cardona, 2016). Esta generación es testigo del desarrollo de las TIC y del procesamiento de grandes volúmenes de información en internet, esto les ha permitido centrar su búsqueda en recomendaciones de personas de su círculo social, amigos y personajes referentes que aparecen en diversas plataformas digitales (Castro et al., 2018).

En cuando al comercio electrónico y compras por internet, los millennials en Latinoamérica han superado a los individuos de otras generaciones, lo que significa un componente esencial en la operación de las empresas turísticas (Valdez-Roca, 2018). La llamada generación Y está altamente influenciada por los desarrollos tec- 
nológicos, es el primer segmento de población con un estilo de vida basado en la digitalización de los servicios; además, las redes sociales se han vuelto una herramienta eficaz para buscar, compartir, colaborar y consumir todo tipo de información utilizada en la toma de decisiones (Alan y Kabadayi, 2016; Liberato et al., 2018).

Al considerar a los millennials en el sector de nativos digitales como un segmento de la población más joven (OIJ, 2017), se esperaría que realizaran una cantidad insignificante de viajes por negocios; sin embargo, en países como Estados Unidos representan al menos un tercio del desplazamiento por este motivo (Valdez-Roca, 2018). Por consiguiente, resulta de interés conocer los factores que les conducen a seleccionar los destinos, considerando que cumplen con las expectativas indispensables para solventar sus necesidades básicas durante la estancia, al cumplir una agenda empresarial o por vacaciones (Jividen, 2017).

No obstante, el comportamiento de los millennials no es sencillo de predecir, su patrón de decisión puede cambiar por algún suceso de última hora, comentario o punto de vista de alguna persona a la que siguen en internet, la atención al cliente y la creación de valor tiene mucho significado para este mercado (Posso y Cardona, 2016). Entre las causas de su comportamiento por priorizar la búsqueda de información y venta en línea, se observa que prefieren la inmediatez que encuentran en internet antes de pasar muchas horas en las tiendas físicas para seleccionar y decidir su compra (Simanjuntak, 2019).

Este segmento de la población permanece mucho tiempo en las redes sociales para intercambiar experiencias, se les identifica por tener un alto nivel de extroversión, que se relaciona con la necesidad de apertura a relacionarse con otros usuarios con los cuales intercambian opiniones sobre temas de actualidad, presentes en los diferentes portales de la web (Alan y Kabadayi, 2016).

Además, se distinguen por establecer un fuerte lazo de amistad con las personas con las que socializan en internet, se preocupan por lo que sus amigos piensan de ellos, lo que se relaciona con escuchar estas opiniones en la toma de decisión referente a los servicios turísticos que consumen (Jividen, 2017). A pesar de que comprar en línea es algo habitual para la generación $Y$, ellos revisan con mucho cuidado el proceso de pago en las plataformas antes de asegurar la transacción; este comportamiento de desconfianza generalmente es por la información obtenida de otros usuarios que comparten sus experiencias de compra en medios digitales (Valdez-Roca, 2018). 
Adicionalmente, la reputación de una empresa como marca en internet es un componente fundamental que condiciona la decisión de compra de los nativos digitales; por tal motivo, el mantenimiento de una estrategia de gestión de relaciones con los clientes en redes sociales se convierte en un medio necesario para las empresas turísticas en la comunicación con los consumidores (Prasad et al., 2019). Es importante que la organización atien da las necesidades y expectativas de este segmento, los millennials son menos leales a las compañías de viajes y hospedaje pues están dispuestos a cambiar de aerolínea u hotel si consideran que otro programa de fidelización ofrece un mejor producto en su conjunto (Lee, 2014).

Otra característica de los turistas de la generación $Y$ es que son flexibles al momento de viajar en cuanto a la fecha y destino, su patrón de consumo no es fácil de predecir, no tienen una preferencia específica; sin embargo, la influencia recibida puede derivarse de las vivencias revisadas en los canales de personas influyentes en internet. Además, se inclinan por asegurar tiempo para la diversión durante su estancia en el destino (Carvalho et al., 2015).

Cabe destacar que los millennials son más propensos a utilizar sus millas y puntos canjeables por viajes gratis o con descuentos, en lugar de cobrar una gran cantidad de puntos de recompensa, incluso no encuentran como elemento importante utilizar una marca específica de tarjeta de crédito (Lee, 2014). Con ello, las empresas turísticas deben contemplar diferentes tácticas y estrategias dinámicas para ganarse su lealtad y tratar de comprender su comportamiento, siendo que las generaciones que vienen detrás de ellos pueden ser aún más difíciles de predecir, considerando las tendencias actuales.

\section{Metodología}

La investigación que se presenta en este texto es cuantitativa, la cual se distingue por recoger y analizar los datos obtenidos mediante un cuestionario (Cadena-Íñiguez et al., 2017). Con la base de datos analizada en el programa IBM SPSS Statistics 20 se describen los resultados; asimismo, se realiza un análisis factorial para conocer los servicios tecnológicos que utilizan los millennials antes, durante y después del destino visitado. Se optó por atraer como población de estudio a las personas de la generación Y nacidas entre 1981 y el año 2000 (Carvalho et al., 2015; Díaz-Sarmiento 
et al., 2017), residentes de la ciudad de Tijuana, Baja California, los cuales realizaron al menos cinco viajes fuera de su estado en 2019.

La población de ese segmento en dicho municipio es aproximadamente de 1 millón 641 mil 570 habitantes, de los cuales 571 mil 265 tienen entre 20 y 39 años, en correspondencia al rango generacional (Instituto Nacional de Estadística y Geografía, INEGI, 2018). Estos datos fueron la base para adquirir la muestra representativa de 385 individuos a quienes se les aplicó el instrumento, mediante el uso de la fórmula aplicada al tamaño de la población, con $95 \%$ de confianza y $5 \%$ de error admitido (Fischer y Espejo, 2017; Hernández et al., 2014).

La encuesta se realizó en enero y febrero del 2020, mediante un muestreo probabilístico aleatorio simple. La aplicación del cuestionario se efectuó en dos de las principales zonas de mayor afluencia en la ciudad de Tijuana (Zona Río y Centro). Las preguntas filtro fueron que el individuo hubiera realizado al menos cinco viajes fuera del estado de Baja California en el 2019 y tener la edad de entre 20 y 39 años.

Para obtener la información se aplicó un cuestionario que contempló las dimensiones que se presentan en la tabla 1. En la primera sección se inquiere la marca del dispositivo móvil que manejan las personas encuestadas, para conocer los sistemas operativos que utilizan los millennials, el lugar y/o plataforma que usan para comprar el servicio de avión, dónde se hospedaron en el último viaje y el formato recurrido para la reserva de hospedaje. La segunda dimensión se refiere a la frecuencia con que acceden a los servicios tecnológicos durante su viaje.

Asimismo, en el tercer apartado se indaga acerca del medio que utilizan con mayor frecuencia para realizar recomendaciones. En la cuarta sección del instrumento se conoce las plataformas que más usan para comunicarse durante su estancia. En la quinta dimensión se revisa la frecuencia con que utilizan las aplicaciones digitales mientras recorren un destino; además, se evalúa el nivel en que los servicios tecnológicos empleados contribuyen a su experiencia de viaje. Por último, se obtuvieron algunos datos sociodemográficos de la muestra encuestada. 


\section{Tabla 1. Dimensiones del cuestionario aplicado}

\section{Dimensiones}

Tipo de preguntas

\section{Dimensión 1}

Marca del dispositivo móvil; entorno empleado para reservar el servicio de avión; medio que utiliza para informarse del destino; dónde se hospedó; formato para reservar el lugar de hospedaje.

Opción múltiple

\section{Dimensión 2}

Servicios tecnológicos utilizados durante su viaje: guía turístico; comparar lugares de interés; servicio de taxi privado; transporte público; búsqueda de recomendaciones; compartir experiencias en redes sociales; buscar recomendaciones en Google; buscar recomendaciones en redes sociales; buscar información de eventos en el destino; buscar

Escala de Likert información de lugares culturales y recreativos; uso de mapa digital; buscar restaurantes; compartir recomendaciones; consultar el clima.

\section{Dimensión 3}

Medio que utiliza con mayor frecuencia para realizar recomendaciones.

Opción múltiple

\section{Dimensión 4}

Frecuencia con que utiliza las aplicaciones digitales: Facebook; Twitter; Youtube; Instagram; Tripadvisor; Yelp; Hangouts; WhatsApp; Wechat; Snapchat; Tumblr; Pinterest; Netflix; Tinder; Uber.

\section{Dimensión 5}

Contribución de los servicios digitales en la experiencia de viaje: compartir experiencias; eficiencia para desplazarse en el destino; obtener información turística; obtener información de los restaurantes que visita; calificar el servicio de los lugares visitados; utilizar el servicio de taxi privado desde la aplicación de smartphone; comparar destinos de interés; servicios disponibles de compra en línea; consultar el clima durante su estancia.

\section{Dimensión 6}

Información socioeconómica de la persona encuestada: género; nivel de estudios; edad.

Opción múltiple

Fuente: Elaboración propia

En la tabla 2 se presentan los resultados del análisis estadístico de confiabilidad con Alfa de Cronbach, utilizando el programa IBM SPSS Statistics 20. Para este proceso se incluyeron las preguntas presentes en las dimensiones del cuestionario con escala de Likert, al obtener valores por encima de 0.7 se tiene consistencia interna (Hinton et al., 2014) lo que se traduce en buena confiabilidad para el instrumento aplicado en la investigación.

\section{Tabla 2. Resultados de estadísticas de confiabilidad con Alfa de Cronbach}

\begin{tabular}{|c|c|c|c|c|c|}
\hline \multicolumn{2}{|c|}{$\begin{array}{c}\text { Servicios tecnológicos } \\
\text { utilizados durante su viaje }\end{array}$} & $\begin{array}{c}\text { Frecuencia con que utiliza las } \\
\text { apps de internet }\end{array}$ & $\begin{array}{c}\text { Contribución de los servicios } \\
\text { digitales en la experiencia de viaje }\end{array}$ \\
\hline Alfa de Cronbach & $\begin{array}{c}\mathrm{N} \text { de } \\
\text { elementos }\end{array}$ & Alfa de Cronbach & $\begin{array}{c}\mathrm{N} \text { de } \\
\text { elementos }\end{array}$ & Alfa de Cronbach & $\begin{array}{c}\mathrm{N} \text { de } \\
\text { elementos }\end{array}$ \\
\hline 0.928 & 13 & 0.839 & 16 & 0.953 & 9 \\
\hline
\end{tabular}

Fuente: Elaboración propia 
Para la gestión de los datos obtenidos se optó por la estadística descriptiva, con base en las preguntas realizadas en el cuestionario aplicado a los viajeros millennials, con la finalidad de revisar las tendencias en el uso de los servicios digitales durante el viaje. La segunda sección de resultados corresponde al análisis factorial.

\section{Resultados}

Con base en las respuestas, los equipos smartphone que más utilizan los viajeros de la generación $Y$ encuestados corresponden al siguiente orden: $43.4 \%$ cuentan con iPhone; $29.9 \%$ disponen de equipo de la marca Samsung; $8.8 \%$ Huawei; $6.2 \%$ posee Motorola; $4.4 \%$ tiene $L G$ y el $6 \%$ restante se concentra en dispositivos de las marcas Sony, Xiaomi, ZTE, Lenovo, HTC, Alcatel y Lanix.

En cuanto al medio para reservar transporte aéreo (gráfica 1), prefieren acceder al servicio disponible desde el sitio web de las aerolíneas (57.1\%), seguido de usar la app del dispositivo móvil (16.6\%); asimismo, el 10.6\% antepone acudir a la agencia de viaje física. El 9.6\% dispone de páginas intermediarias (Kayak, Bestday, Expedia, etcétera) y $6 \%$ prefiere llamar por teléfono a la compañía.

Estos resultados se relacionan con el perfil de estudio, según datos de Villar-Lama (2018), Gustavo (2013) y la OMT (2016), en los que se afirma que los jóvenes utilizan su smartphone para múltiples consultas en páginas web y apps turísticas a fin de obtener información respecto a su viaje. Asimismo, mediante la marca de los dispositivos móviles se puede conocer el sistema operativo que utilizan, para que las empresas turísticas puedan crear sus apps compatibles con esos sistemas operativos. 
Gráfica 1. Medio que utiliza para reservar el servicio de avión

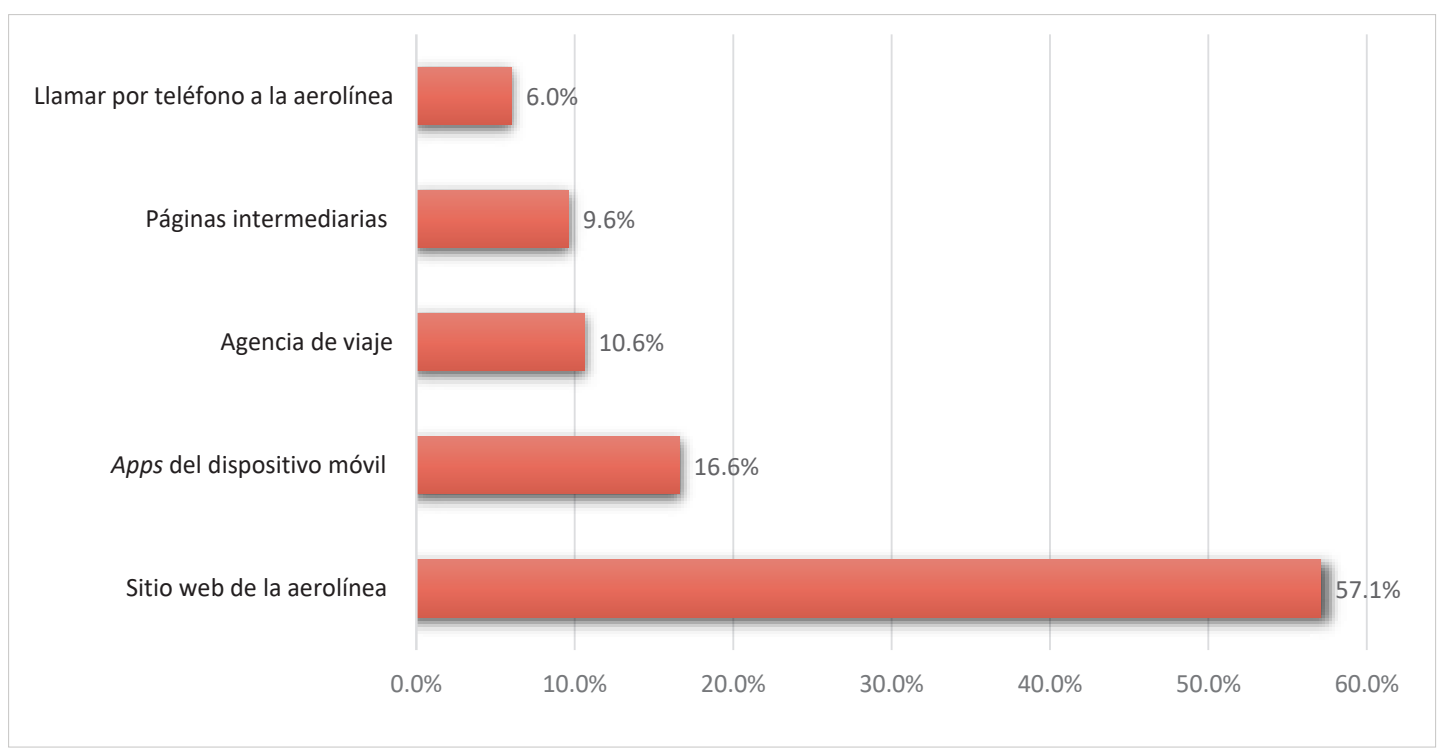

Fuente: Elaboración propia.

En cuanto al lugar donde se hospedaron en su último viaje (gráfica 2), 40.5\% de la muestra optó por quedarse en hotel; $34.5 \%$ llegaron con familiares o amigos; $13.8 \%$ rentaron departamento; $8.3 \%$ eligieron rentar casa; $1.6 \%$ prefirieron utilizar el servicio de hostal y solamente el $1.3 \%$ reservaron una cabaña. Cabe destacar que el $65.5 \%$ de los encuestados utilizaron algún medio tecnológico para la reserva de hospedaje. Con base en este resultado, los viajeros con perfil millennial dispusieron principalmente de los canales del hotel para reservar su alojamiento, seguido del sistema de la plataforma Airbnb, además de los servicios de intermediarias como Trivago, Booking, Expedia, Kayak, Hotel Tonight, entre otros.

Es de resaltar la necesidad de este segmento de la población de recurrir a los canales necesarios para anticipar su experiencia, teniendo como principal aliado a los medios digitales. De acuerdo con Macanás-López (2019), para ellos la tecnología se ha convertido en un factor para la experiencia de viaje, buscan información en internet y también comparan ofertas de hospedajes y de aerolíneas (Lee, 2014). 


\section{Gráfica 2. Lugar de hospedaje}

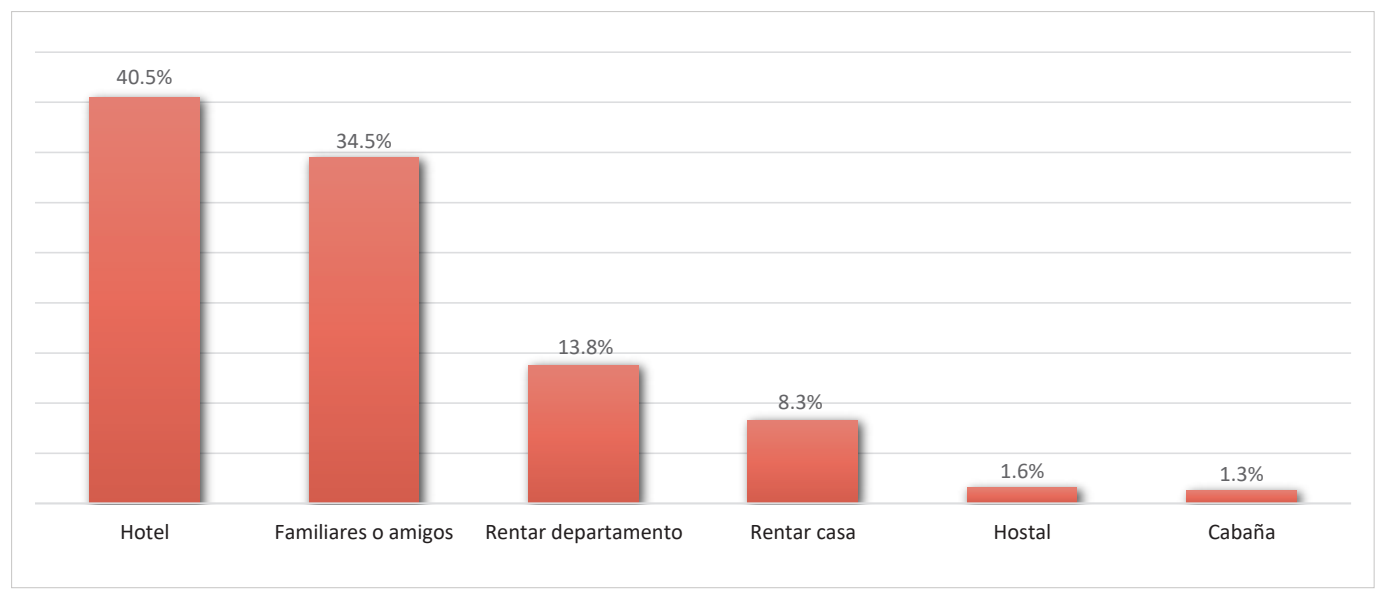

Fuente: Elaboración propia.

Entre las herramientas digitales más utilizadas para compartir experiencias durante el viaje se observa con mayor porcentaje la opción de Facebook (42.1\%), otra proporción importante prioriza esta actividad mediante Instagram (18.2\%), seguido de Google (14.8\%) que se caracteriza por permitir la aportación de comentarios y calificar los diversos lugares visitados (gráfica 3). Resalta que $17.1 \%$ de los encuestados omite compartir su experiencia durante el viaje. Las aplicaciones para viajeros entre las que se encuentran Yelp y Tripadvisor no son las más importantes para este segmento de la población. Estos resultados concuerdan con Alan y Kabadayi (2016) y Melián y Bulchand (2015) en los cuales la generación Y comparte sus experiencias de viajes en las redes sociales y sitios web de los negocios del sector turístico.

Gráfica 3. Medio que utiliza para realizar recomendaciones

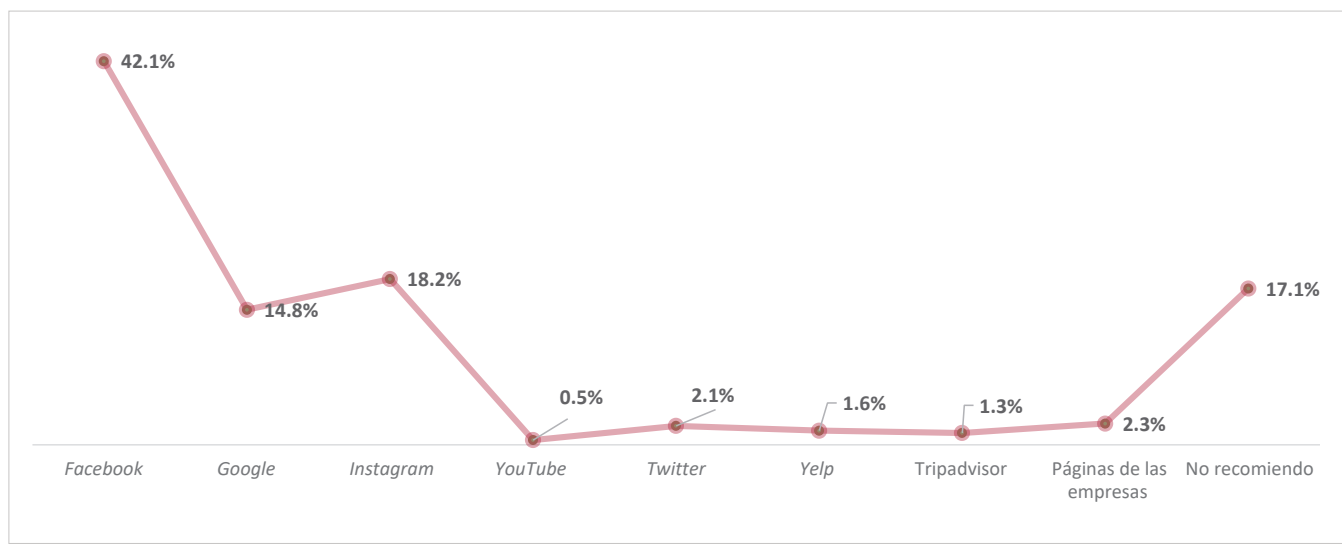

Fuente: Elaboración propia 
Se preguntó igualmente la frecuencia de uso, en una escala del 1 al 5, con base en la incidencia en que utilizan los servicios tecnológicos. En este caso se destacan cinco principales (gráfica 4): con mayor importancia se presenta el uso del sistema de geolocalización, que funciona para optimizar el recorrido del visitante y evitar las zonas con mayor aglomeración. También se destaca la consulta del clima, que sirve para anticiparse a las situaciones que puedan ocurrir durante la semana, del mismo modo la búsqueda de restaurantes resulta ser un componente empleado con frecuencia, permitiendo conocer recomendaciones y la calificación de otros consumidores.

El servicio de taxi privado por sistema o aplicación de internet también resulta fundamental, por la seguridad de la persona y movilidad en la ciudad. Finalmente, se integra el compartir experiencias en redes sociales, siendo uno de los hábitos practicados por los turistas de la actualidad. Con ello, se concuerda con la OIJ (2017) respecto a que la conectividad tecnológica es central en la vida de la generación Y.

Gráfica 4. Servicios tecnológicos más utilizados durante el viaje

Recomendar lugares visitados
Utilizar servicio de
geolocalización
Información de lugares culturales
y recreativos
Información de eventos en el
destino

En cuanto a las apps instaladas en el smartphone empleadas durante el viaje, se preguntó la frecuencia de uso en una escala del 1 al 5, con base en la frecuencia con que las utilizan. En los resultados se anteponen las herramientas de redes sociales y comunicación, Facebook, WhatsApp e Instagram (gráfica 5). Otras aplicaciones que pueden 
considerarse con acceso importante son el servicio de taxi privado (Uber) y consulta de YouTube, que se caracteriza por presentar videos que pueden resultar de entretenimiento y apoyo de material audiovisual, relacionado con los intereses de los usuarios de este perfil. Para Alan y Kabadayi (2016) y Liberato et al. (2018) el uso de los servicios digitales es un estilo de vida para los millennials, en los resultados encontrados estos jóvenes usan las apps para todos los servicios que utilizan en su viaje.

\section{Gráfica 5. Apps utilizadas durante el viaje}

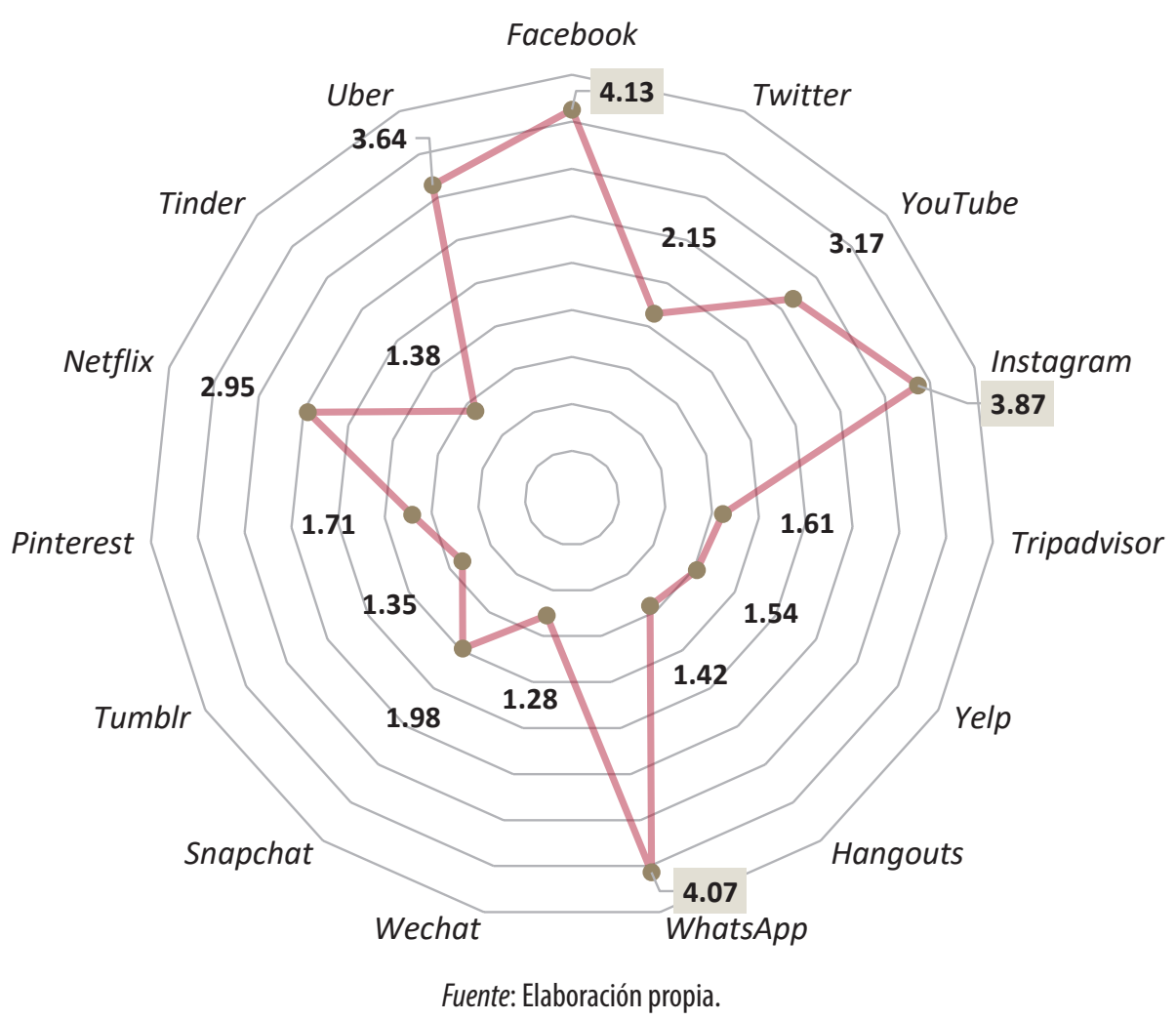

En las siguientes líneas se presenta el análisis factorial realizado para conocer los servicios tecnológicos que utilizan antes, durante y después del destino visitado, que benefician su experiencia de viaje. Con base en Pérez (2004), el valor KaiserMeyer-Olkin (KMO) superior a 0.5 se considera aceptable en el modelo de análisis factorial, mientras más cerca esté de 1 mejor es la adecuación de los datos. Al obtener 0.915 significa que los datos pueden ser utilizados para la exposición factorial (tabla 3). El coeficiente de esfericidad de Bartlett derivado del proceso indica que existe correlación entre las variables, esto permite validar el procedimiento de análisis factorial, ya que el nivel de significancia derivado es menor de 0.05 . 
Tabla 3. Valor KMO y prueba de esfericidad Bartlett

\begin{tabular}{|l|l|r|}
\hline Medida Kaiser-Meyer-Olkin de adecuación de muestreo & .915 \\
\hline & Aprox. Chi-cuadrado & 2383.851 \\
\hline Prueba de esfericidad de Bartlett & gl & 45 \\
\hline & Sig & 0.000 \\
\hline
\end{tabular}

Fuente: Elaboración propia

En lo que corresponde a la matriz de correlaciones anti-imagen, De la Garza et al. (2013) afirman que proporciona una medida de relación entre las variables, los valores de medida de suficiencia o adecuación del muestreo general (MSA) deben ser mayores a 0.5 ; si estos valores son menores a este parámetro deben ser eliminados del estudio. Por lo tanto, una vez realizada la depuración de los elementos que no cumplían con el parámetro establecido se logró el resultado que se presenta en la tabla 4.

Tabla 4. Correlaciones - matriz anti-imagen

\begin{tabular}{|c|c|c|c|c|c|c|c|c|c|c|c|}
\hline \multicolumn{12}{|c|}{ Matrices anti-imagen } \\
\hline & & 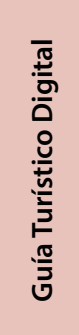 & 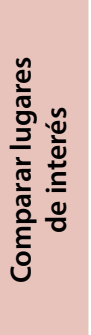 & 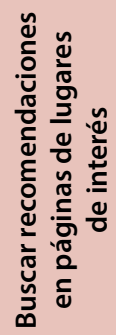 & 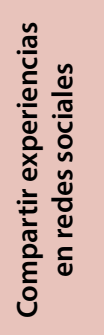 & 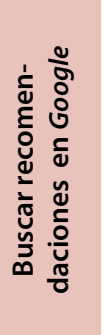 & 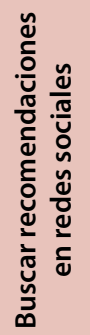 & 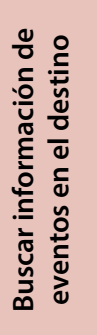 & 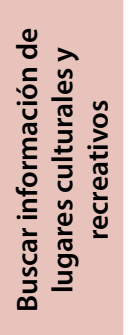 & 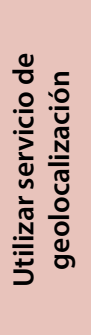 & 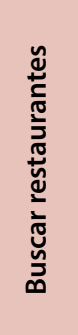 \\
\hline \multirow{10}{*}{ 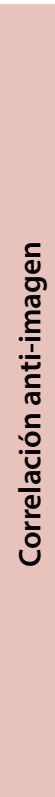 } & Guía Turístico Digital & $.769^{\mathrm{a}}$ & -.440 & .078 & .096 & -.004 & .008 & -.184 & -.091 & .109 & -.015 \\
\hline & $\begin{array}{c}\text { Comparar lugares de } \\
\text { interés }\end{array}$ & -.440 & $.882^{\mathrm{a}}$ & -.149 & -.112 & -.006 & .040 & -.016 & -.023 & -.114 & -.059 \\
\hline & $\begin{array}{c}\text { Buscar } \\
\text { recomendaciones en } \\
\text { páginas de lugares de } \\
\text { interés }\end{array}$ & .078 & -.149 & $.937^{\mathrm{a}}$ & -.235 & -.304 & -.051 & -.098 & -.128 & -.026 & -.062 \\
\hline & $\begin{array}{l}\text { Compartir experiencias } \\
\text { en redes sociales }\end{array}$ & .096 & -.112 & -.235 & $.942^{\mathrm{a}}$ & -.129 & -.202 & -.013 & .000 & -.061 & .000 \\
\hline & $\begin{array}{l}\text { Buscar } \\
\text { recomendaciones } \\
\text { en Google }\end{array}$ & -.004 & -.006 & -.304 & -.129 & $.918^{\mathrm{a}}$ & -.255 & -.110 & .098 & -.260 & -.148 \\
\hline & $\begin{array}{l}\text { Buscar } \\
\text { recomendaciones en } \\
\text { redes sociales }\end{array}$ & .008 & .040 & -.051 & -.202 & -.255 & $.923^{\mathrm{a}}$ & -.224 & -.176 & .166 & -.119 \\
\hline & $\begin{array}{l}\text { Buscar información de } \\
\text { eventos en el destino }\end{array}$ & -.184 & -.016 & -.098 & -.013 & -.110 & -.224 & $.923^{\mathrm{a}}$ & -.395 & -.028 & -.023 \\
\hline & $\begin{array}{c}\text { Buscar información } \\
\text { de lugares culturales y } \\
\text { recreativos }\end{array}$ & -.091 & -.023 & -.128 & .000 & .098 & -.176 & -.395 & $.911^{\mathrm{a}}$ & -.234 & -.201 \\
\hline & $\begin{array}{l}\text { Utilizar servicio de } \\
\text { geolocalización }\end{array}$ & .109 & -.114 & -.026 & -.061 & -.260 & .166 & -.028 & -.234 & $.905^{\mathrm{a}}$ & -.318 \\
\hline & Buscar restaurantes & -.015 & -.059 & -.062 & .000 & -.148 & -.119 & -.023 & -.201 & -.318 & $.942^{\mathrm{a}}$ \\
\hline
\end{tabular}


Asimismo, en la tabla 5 se muestra el resultado del porcentaje de la varianza total que se resume a cinco factores, los cuales explican el $68.995 \%$ para este caso. De la Garza et al. (2013) señala que mediante este criterio se considera que $n$ factores deben manejarse como solución inicial, siempre y cuando el porcentaje de variación explicada acumulada se encuentre en un rango de entre 60 a $95 \%$.

Tabla 5. Varianza total explicada

\begin{tabular}{|c|c|c|c|c|c|c|c|c|c|}
\hline \multirow{2}{*}{ Componente } & \multicolumn{3}{|c|}{ Autovalores iniciales } & \multicolumn{3}{|c|}{$\begin{array}{c}\text { Sumas de extracción de cargas al } \\
\text { cuadrado }\end{array}$} & \multicolumn{3}{|c|}{$\begin{array}{l}\text { Sumas de rotación de cargas } \\
\text { al cuadrado }\end{array}$} \\
\hline & Total & $\begin{array}{c}\% \text { de } \\
\text { varianza }\end{array}$ & $\begin{array}{c}\% \\
\text { acumulado }\end{array}$ & Total & $\begin{array}{c}\% \text { de } \\
\text { varianza }\end{array}$ & $\begin{array}{c}\% \\
\text { acumulado }\end{array}$ & Total & $\begin{array}{c}\% \text { de } \\
\text { varianza }\end{array}$ & $\begin{array}{c}\% \\
\text { acumulado }\end{array}$ \\
\hline 1 & 5.787 & 57.873 & 57.873 & 5.787 & 57.873 & 57.873 & 5.021 & 50.208 & 50.208 \\
\hline 2 & 1.112 & 11.122 & 68.995 & 1.112 & 11.122 & 68.995 & 1.879 & 18.788 & 68.995 \\
\hline ... & & & & & & & & & \\
\hline 10 & .204 & 2.037 & 100.000 & & & & & & \\
\hline
\end{tabular}

Método de extracción: análisis de componentes principales

\section{Fuente: Elaboración propia}

En seguimiento al procedimiento de análisis factorial, se muestra la matriz de componentes rotados, para el que se utilizó el método Varimax (tabla 6). Los autores De la Garza et al. (2013) y Pérez (2004) destacan que mediante este método es posible identificar a un grupo de variables con un solo factor simplificado por componente. Por lo tanto, los servicios que utilizan los millennials antes, durante y después de su viaje, que favorecen su experiencia son: 1) acceso a información y servicios durante su estancia; y 2) análisis de la experiencia anticipada.

Tabla 6. Matriz de componentes rotados

\begin{tabular}{|l|c|c|} 
& \multicolumn{3}{|c|}{ Componente } \\
\hline Guía turístico digital & 1 & 2 \\
\hline Comparar lugares de interés & .061 & .924 \\
\hline Buscar recomendaciones en páginas de lugares de interés & .381 & .727 \\
\hline Compartir experiencias en redes sociales & .817 & .195 \\
\hline Buscar recomendaciones en Google & .754 & .063 \\
\hline Buscar recomendaciones en redes sociales & .847 & .161 \\
\hline Buscar información de eventos en el destino & .779 & .183 \\
\hline Buscar información de lugares culturales y recreativos & .733 & .404 \\
\hline Utilizar servicio de geolocalización & .761 & .368 \\
Buscar restaurantes & .763 & .185 \\
\multicolumn{1}{|c|}{ Método de extracción: análisis de componentes principales. } & .248 \\
\hline Método de rotación: Varimax con normalización Kaisera. & \\
\hline \multicolumn{1}{|c|}{ a. La rotación ha convergido en tres iteraciones. } &
\end{tabular}

Fuente: Elaboración propia 
Como se mencionó previamente, resulta importante conocer los servicios tecnológicos que usan los viajeros millennials, ya que estos se transforman en canales de comunicación para hacer llegar la oferta turística a ese sector de la población (Orgaz y Moral, 2016). Asimismo, el uso de las TIC tiene un lugar primordial que contribuye a la experiencia positiva del turista antes, durante y después del viaje (Amadeus, 2015).

\section{Discusión}

Los turistas que corresponden al segmento referido tienen una amplia inclinación por las compras en línea comparado con las generaciones que le preceden (Valdez-Roca, 2018); además, la generación Y está altamente influenciada por los desarrollos tecnológicos, el uso de los servicios digitalizados es su estilo de vida (Alan y Kabadayi, 2016; Liberato et al., 2018), comparten sus experiencias de viaje en las redes sociales y buscan información en las páginas web de los negocios turísticos (Alan y Kabadayi, 2016; Melián y Bulchand, 2015).

En los resultados obtenidos, los millennial prefieren la inmediatez y accesibilidad que le proporcionan los servicios tecnológicos para comprar, comparar precios, realizar comentarios en los sitios web de los negocios turísticos y en las redes sociales. Cabe destacar que los consumidores de esta generación no están acostumbrados a pasar muchas horas en tiendas físicas para seleccionar su compra, prefieren agilizar este proceso mediante el uso de las tecnologías (Simanjuntak, 2019).

Este perfil turístico busca comparar precios en internet, realizar múltiples consultas para obtener información de sus viajes, comprar en línea, hacer recomendaciones en las redes sociales, blogs y páginas web. Durante todo su viaje están conectados a internet desde su smartphone (Villar-Lama, 2018; Gustavo, 2013; OMT, 2016).

En esta investigación, la red social que más utilizan los encuestados para realizar recomendaciones es Facebook, compran sus boletos de avión desde la página web de las aerolíneas, se hospedan en hoteles o con familiares y amigos, realizan búsquedas de información por Google; las apps que usan con mayor frecuencia son $\mathrm{Fa}$ cebook, Instagram, WhatsApp, Uber, YouTube; finalmente, los servicios tecnológicos que más utilizan durante sus viajes son: buscar restaurantes, consultar el clima y servicios de geolocalización. 
Para Orgaz y Moral (2016), es importante conocer los servicios tecnológicos que utilizan estos viajeros pues se han convertido en canales de comunicación entre los clientes potenciales y los negocios turísticos. El uso de las TIC en el mercado turístico es una tendencia positiva para las empresas (Cerezo y Guevara, 2015); además, la tecnología contribuye de manera positiva a la experiencia del viajero (Amadeus, 2015). En este sentido, se realizó el análisis factorial para conocer los servicios tecnológicos que usan los jóvenes tijuanenses con este perfil que benefician su experiencia de viaje y se encontraron dos componentes que se engloban en el acceso inmediato a la información y servicios del destino, así como la experiencia anticipada.

\section{Conclusiones}

Como se señaló en este trabajo, los viajeros con perfil millennial residentes de Tijuana prefieren reservar sus vuelos a través del sitio de internet de las aerolíneas, seguido de las apps instaladas en su dispositivo móvil. Para los que optan por hacer uso del servicio de hospedaje en los destinos que visitan, recurren principalmente al sitio web para reservas del hotel, seguido del sistema que oferta alojamientos particulares y turísticos Airbnb, además de las plataformas tecnológicas que funcionan como intermediarios en el servicio de alojamiento (Trivago, Booking, Expedia, Kayak, etcétera).

En cuanto al proceso para enterarse de los servicios y opciones disponibles en el destino, los viajeros de esta generación utilizan principalmente el motor de búsquedas instalado en Google, también optan por preguntar a sus familiares y amigos de sus experiencias obtenidas, además de revisar la información disponible en la red social Facebook.

Una de las características documentadas que distingue a los millennials es el uso de la tecnología como un estilo de vida y la flexibilidad en cuanto a la forma y momento de viajar, su patrón de consumo es complejo de predecir y fidelizar; por lo tanto, se observa como necesario para las empresas turísticas mantener diversos canales de comunicación activos, hacia el desarrollo de una estrategia de entrega de valor en el servicio, con el objetivo de anticiparse a las tendencias que sigue este segmento de la población.

Con relación a compartir su experiencia durante el viaje en los medios digitales, esta actividad la realizan con mayor frecuencia a través de Facebook, además de 
Instagram y el sistema de recomendaciones Google. Cabe destacar que las redes sociales distinguidas para viajeros (Yelp y Tripadvisor) no son las preferidas en este propósito. Aunado a lo anterior, las aplicaciones que tienen mayor actividad durante su travesía en el destino coinciden con Facebook e Instagram y se añade la herramienta de mensajería instantánea WhatsApp, esta última ha tenido una evolución favorable, hasta ser considerada en la categoría de red social.

Es importante destacar los servicios tecnológicos que favorecen la experiencia turística de estos viajeros, como es el uso de las redes sociales en general, la búsqueda de información en los sitios web y redes de los negocios turísticos, consultar las páginas web de los destinos visitados, lugares culturales y recreativos, así como el uso del servicio de la geolocalización. Lo anterior evidencia que las TIC se han convertido en un componente clave dentro de los elementos que hacen competitivo a un destino.

\section{Referencias}

Alan, A. K., y Kabadayi, E. T. (2016). The effect of personal factors on social media usage of young consumers. Procedia - Social and Behavioral Sciences, 235, 595-602. https://doi.org/10.1016/j.sbspro.2016.11.086

Amadeus. (2015). El viajero experto: tres tendencias importantes en el sector de los viajes que se están acelerando con la recesión. https://bit.ly/3oNOPxe

Arnould, E. J. y Price, L. (1993). River magic: extraordinary experience and the extended services encounter. Journal of Consumer Research, 20(1), 24-45. http://dx. doi.org/10.1086/209331

Ballesteros, B., Tavera, J. F. y Castaño, D. (2014). Aceptación tecnológica de la publicidad en dispositivos móviles en Colombia. Semestre Económico, 17(36), 133-153. https://doi.org/10.22395/seec.v17n36a6

Carballo-Fuentes, R. (2014). Experiencia turística, imagen, seguridad y centros culturales [tesis de doctorado, Universidad de las Palmas de Gran Canarias]. Repositorio institucional. https://bit.ly/34mrzek

Caballero, M. y Baigorri, A. (2013). ¿Es operativo el concepto de generación? Aposta, Revista de Ciencias Sociales, 56, 1-45. https://bit.ly/3oQmNzf 
Cadena-Îniguez, P., Rendón-Medel, R., Aguilar-Ávila, J., Salinas-Cruz, E., De la Cruz-Morales, F. y Sangerman-Jarquín, D. (2017). Métodos cuantitativos, métodos cualitativos o su combinación en la investigación: un acercamiento en las ciencias sociales. Revista Mexicana de Ciencias Agrícolas, 8(7), 1603-1617. https://bit.ly/2SpJEFO

Carvalho, J., De Oliveira, M., Do Nascimento, B. y Prudêncio, J. (2015). Carrera, renta y consumo bajo la perspectiva del joven de la generación "Y". Invenio, 18(34), 119-135. https://bit.ly/34ff1pn

Castro, D., Peñaloza, L. yTamayo, A. (2018).Tecnologías en línea populares para viajar: ¿cuáles utilizan los jóvenes universitarios para hacer turismo? Actualidades Investigativas en Educación, 18(2). https://doi.org/10.15517/aie.v18i2.33149

Cerezo, A. y Guevara, A. (2015). Necesidades formativas tecnológicas en turismo. Estudio de caso de Andalucía. PASOS, Revista de Turismo y Patrimonio Cultural, 13(4), 913-929. https://bit.ly/34iuViG

Chang, S. E. y Shen, W. (2018.). Exploring smartphone social networking services for mobile tourism. International Journal of Mobile Communications, 16(1), 63-81. https://doi.org/10.1504/IJMC.2018.10007781

Díaz-Sarmiento, C., López-Lambraño, M. y Roncallo-Lafont, L. (2017). Entendiendo las generaciones: una revisión del concepto, clasificación y características distintivas de los Baby Boomers, XY Millennials. Clío América, 11(22), 188-204. https://doi.org/10.21676/23897848.2440

De la Garza, J., Morales, B. N. y González, B. A. (2013). Análisis estadístico multivariante: un enfoque teórico y práctico. McGraw-Hill.

Feixa, C., Fernández-Planells, A. y Figueras-Maz, M. (2016). Generación hashtag. Los movimientos juveniles en la era de la web social. Revista Latinoamericana en Ciencias Sociales, Niñez y Juventud, 14(1), 107-120. http://dx.doi. org/10.11600/1692715x.1416301115

Fischer, L. y Espejo, J. (2017). Introducción a la investigación de mercados. McGraw-Hill.

Garzaniti, R. (2018). La inteligencia a través de las generaciones: millennials y centennials. Acta de Investigación Psicológica, 8(2), 90-100. https://doi. org/10.22201/fpsi.20074719e.2018.2.08

González-Damián, A. (2018). Construcción social de la experiencia turística. Bubok Publishing S.L. 
Gustavo, N. (2013). Marketing management trends in tourism and hospitality industry: facing the 21 st century environment. International Journal of Marketing Studies, 5(3), 13-25. https://doi.org/10.5539/ijms.v5n3p13

Harrison, J. (2001). Thinking about tourists. International Sociology, 16, 159-172. https://doi.org/10.1177/0268580901016002002

He, Z., Yang, L. y Wei, G. (2014). A day-to-day route choice model based on travellers' behavioural characteristics. Procedia-Social and Behavioral Sciences, 138, 738 - 747. https://doi.org/10.1016/j.sbspro.2014.07.251

Hernández, R., Fernández, C. y Baptista, P. (2014). Metodología de la Investigación. McGraw-Hill.

Hinton, P., McMurray, I. y Brownlow, C. (2014). SPSS Explained. Routledge.

Holbrook, M. B. y Hirschman, E. C. (1982). The experiential aspects of consumption: Consumer fantasies, feelings, and fun. Journal of Consumer Research, 9(2), 132-140. https://doi.org/10.1086/208906

Instituto Nacional de Estadística y Geografía (INEGI). (2018). Anuario estadístico y geográfico por entidad federativa 2018 - México. https://bit.ly/3oO6vHI

Ivars, J., Solsona, F. y Giner, D. (2016). Gestión turística y tecnologías de la información y la comunicación (TIC): el nuevo enfoque de los destinos inteligentes. Documents d'Anàlisi Geogràfica, 62(2). 327-346. http://hdl.handle. net/10045/54852

Jividen, A. (2017). The incredible shrinking space: a small glimpse into the minds of millennial travelers. En D. Edmonson y A. Williams (Eds.), Ethical decisions in lifestyle choices (pp. 428-429). Society for Marketing Advances. https://bit. ly/3wzv79j

Lee, T. (2014). Segmenting millennial travelers business vs leisure. HVS. https://bit. ly/3vojnq6

Liberato, P., Liberato, D., Abreu, A., Alén-González, E. y Rocha, Á. (2018). Generation $\mathrm{Y}$ : the competitiveness of the tourism sector based on digital technology. En T. Antipova y A. Rocha (Eds.), Advances in Intelligent Systems and Computing, Vol. 724. Springer, Cham. https://doi.org/10.1007/978-3-319-74980-8_21

López-Rodríguez, A. y López-Rodríguez, S. (2018). Impacto de las TIC en el turismo: caso colombiano. Cuadernos de Turismo, 41, 399-418. https://doi. org/10.6018/turismo.41.327081 
Macanás-López, C. (2019). Estudio y análisis del comportamiento turístico en perspectiva generacional [tesis de licenciatura, Universidad Politécnica de Valencia]. Repositorio institucional. http://hdl.handle.net/10251/130196

MacCannell, D. (1973). Staged authenticity: arrangements of social space in tourist settings. The American Journal of Sociology, 79(3), 589-603. https://doi. org/10.1086/225585

Mazarrasa, K. (20116). Experiential and creative tourism: the case of Cantabria. International Journal of Scientific Management and Tourism, 2(3), 195-203. https://bit.ly/3vzokw4

Melián, S. y Bulchand, J. (2015). Segunda economía en el sector turístico:TIC y puestos de trabajo. PASOS, Revista de Turismo y Patrimonio Cultural, 13(5), $1265-$ 1275. https://doi.org/10.25145/j.pasos.2015.13.085

Neuhofer, B., Buhalis, D. y Ladkin, A. (2013). A typology of technology-enhanced tourism experiences. International Journal of Tourism Research, 16, 340-350. https://doi.org/10.1002/jtr.1958

Organismo Internacional de Juventud para Iberoamérica (OIJ). (2017). Millenials ¿Una categoría útil para identificar a las juventudes Iberoamericanas? https://bit.ly/3uvlhUJ

Ordaz-Mejía, D. A. y Osorio-García, M. (2016). Significados del viaje turístico en jóvenes millennials. Caso de estudio: Texcoco, México. Revista Latinoamericana de Ciencias Sociales, Niñez y Juventud, 16(2), 897-912. https://doi.or$\mathrm{g} / 10.11600 / 1692715 \mathrm{x} .16217$

Organización Mundial del Turismo (OMT). (2016). Global report on the power of youth travel. En Equipo Editorial OMT (Eds.), Affiliate Members Global Report, Vol. 13. https://bit.ly/3ujYivp

Orgaz, F. y Moral, S. (2016). El turismo como motor potencial para el desarrollo económico de zonas fronterizas en vías de desarrollo. Un estudio de caso. El Periplo Sustentable, 31. http://dx.doi.org/10.21854/eps.v0i31.2477

Pérez, C. (2004). Técnicas de Análisis Multivariante de Datos. Pearson

Posso, Á. y Cardona, D. (2016). Tendencias de consumo turístico de los millennials en la ciudad de Ibarra. Ecos de la Academia, 2(4), 145-157. https://bit.ly/3oPWBVy 
Prasad, S., Garg, A. y Prasad, S. (2019). Purchase decision of generation Y in an online environment. Marketing Intelligence and Planning, 37(4), 372-385. https://doi. org/10.1108/MIP-02-2018-0070

Ralph, K. M. (2017). Multimodal millennials? The four traveler types of young people in the United States in 2009. Journal of Planning Education and Research, 37(2), 150-163. https://doi.org/10.1177/0739456X16651930

Rivera, M. (2013). El turismo experiencial como forma de turismo responsable e intercultural. En L. Rodríguez y A. Roldán (Eds.) Relaciones interculturales en la diversidad (pp. 199-217). Universidad de Córdoba. https://bit.ly/3vqVAG6

Rodríguez-Moreno, D. (2018). Tecnologías de información y comunicación para el turismo inclusivo. Revista Facultad de Ciencias Económicas, 26(1), 125-146. https://doi.org/10.18359/rfce.3142

Ruiz-Cartagena, J. J. (2017). Millennials y redes sociales: estrategias para una comunicación de marca efectiva. Miguel Hernández Communication Journal, 12(8), 347-368. https://doi.org/10.21134/mhcj.v0i8.196

Simanjuntak, M. (2019). Generation Y's complaint behavior toward online shopping. Independent Journal of Management and Production, 10(1), 101. https://doi. org/10.14807/ijmp.v10i1.839

Tung, V. W. y Brent, J. R. (2011). Exploring the essence of memorable tourism. Annals of Tourism Research, 38(4), 1367-1386. https://doi.org/10.1016/j. annals.2011.03.009

Ulloa, C. (Ed.). (2018). Los millennials frente al espejo: tecnología, jóvenes y educación. Universidad de las Américas, Colección -logía. https://bit.ly/3hUdcpY

Valdez-Roca, A. F. (2018). Un mercado potencial: Perfil turístico de los estudiantes de administración en turismo y hotelería de la Universidad César Vallejo (Lima, Perú). Estudios y Perspectivas en Turismo, 27(3), 756-782. https://bit. ly/3yELK5h

Vieira, A., Mendes-Filho, L. y Do Nascimento, A. (2017). Evaluación de la información de una aplicación turística: un análisis realizado por profesionales del turismo sobre la e-Guía Find Natal (Brasil). Estudios y Perspectivas en Turismo, 26(4), 884-904. https://bit.ly/34je2V6 
Villar-Lama, A. (2018). Ocio y turismo millennial: el fenómeno de las salas de escape. Cuadernos de Turismo, 41, 615-636. https://doi.org/10.6018/ turismo.41.327181 